AperTO - Archivio Istituzionale Open Access dell'Università di Torino

\title{
Radiation awareness in an Italian multispecialist sample assessed with a web-based survey
}

\section{This is the author's manuscript}

Original Citation:

Availability:

This version is available http://hdl.handle.net/2318/1739200

since 2020-05-20T16:24:14Z

Published version:

DOI:10.1080/00015385.2020.1733303

Terms of use:

Open Access

Anyone can freely access the full text of works made available as "Open Access". Works made available under a Creative Commons license can be used according to the terms and conditions of said license. Use of all other works requires consent of the right holder (author or publisher) if not exempted from copyright protection by the applicable law. 
Radiation awareness in an Italian multispecialist sample assessed with a web-based survey

Marzia Giaccardi MD ${ }^{1}$; Matteo Anselmino $\mathrm{MD}^{2}$, Giuseppe Mascia MD, $\mathrm{PhD}^{1}$, Maurizio Del Greco $\mathrm{MD}^{3}$, Gaetano Maria De Ferrari MD², Eugenio Picano MD

${ }^{1}$ Cardiology and Electrophysiology Unit, Department of Internal Medicine, Azienda USL Toscana Centro, Florence, Italy.

${ }^{2}$ Division of Cardiology, “Città della Salute e della Scienza di Torino” Hospital, Department of Medical Sciences, University of Turin, Italy

${ }^{3}$ Cardiology Rovereto Hospital APSS Trento. Italy

CNR Institute of Clinical Physiology, Pisa, Italy

Disclosures: All authors report no relationships relevant to the contents of this paper to disclose.

Address for correspondence: Giuseppe Mascia MD, PhD, FESC

e-mail: giuseppe_mascia@virgilio.it ; Mob: +39 3337513936 ; Fax: +39 05569321 


\begin{abstract}
Introduction. The awareness of radiation doses and risks, also during interventional cardiology procedures, is essential today in order to apply the risk-benefit assessment and to reinforce the principles of justification and optimisation in clinical practice.

Methods. A voluntary survey with 10 questions and multiple-choice answers was run on a popular cardiology website (www.cardiolink.it) independently by a scientific publisher, in order to evaluate the contemporary level of radiation awareness in a multi-speciality sample of physicians in Italy.

Results. One thousand eight hundred and sixty-one physicians completed the test. The survey showed good results since both prescribers and practitioners (mostly cardiologists) working in Italy are largely aware of the cancer and non-cancer risks of medical radiation use, regardless of their subspecialty background.

Conclusion. Physicians are largely aware of the cancer and non-cancer risks of medical radiation use, regardless of their subspecialty background. However, there is still broad space for improvement; in the future, the awareness of radiation risk is a prerequisite to create a culture of respect for radiation hazard and a commitment to minimise exposure and maximise protection.
\end{abstract}




\section{Introduction}

Since the introduction in the clinical practice, the ionising radiation marked a turning point in the history of medicine but the increasing use and complexity of imaging and interventional techniques have not been matched by increasing awareness and knowledge by prescribers and practitioners [1]. The awareness of radiation doses and risks is essential in order to apply the risk-benefit assessment and reinforce the principles of justification and optimisation in clinical practice. For this reason, in order to evaluate the contemporary level of radiation awareness in a multi-specialist sample of physicians in Italy, we submitted a survey consisting of 10 questions related to doses and risks of common radiology examinations and basic regulatory framework of radiological practice.

\section{Methods}

A voluntary survey with 10 questions and multiple-choice answers was run on a popular cardiology website (www.cardiolink.it) independently by a scientific publisher in order to address aspects of radiation use in clinical practice. Free registration to website was necessary, such as approval of specific privacy data policy (article 13, 196/2003 of the Italian Regulation and 13, 679/2016 of the European Union, respectively available at https://www.cardiolink.it/index.php?option=com_content\&view $=$ article \&id=15070\&demo $=1$ \&Itemid=42). The survey at first was online on 1 January 2019, ending on 1 March 2019. Among 2023 physicians who started, 1861 completed the 10-step survey.

\section{Study questionnaire}

The questionnaire investigated the level of radiation awareness, focussing on aspects of radiation in clinical practice. Table 1 shows the complete list of questions. In addition, according to the answer of question \#1 (major clinical activity), all physicians were stratified 
exposed (interventional cardiologists: hemodynamic catheter lab, electrophysiology, cardiac stimulation and vascular surgeons) or not exposed (all the other categories).

\section{Statistical analysis}

Continuous variables are reported as median and standard deviation, while categorical variables as number, and percentage (\%). Categorical variables were compared in crosstabulation tables by means of the Fisher chi-square test. All tests of significance were twotailed and a $p$ value $<0.05$ was considered of statistical significance. Analyses were performed using SPSS 21.0 (IBM, Armonk, NY).

\section{Results}

The physicians completing the 10 -step multiple choice survey $(n=1861)$ were mostly cardiologists $(40 \%$ clinical cardiologists, 32\% interventional cardiologists and cardiac electrophysiologists; Table 2). The average time in order to complete the survey was $10 \pm 2$ min. Participants were distributed within all Italian regions, with those from Lombardia and Lazio being the most represented. According to a self-definition of the clinical activity, 484 participants (26\%) were classified as exposed to X-rays while 1377 (74\%) were not professionally exposed. The physicians responsible for most medical irradiations were identified as orthopaedics $(67 \%)$ followed by cardiologists $(24 \%)$ and radiologists $(9 \%)$. This perception was consistent also when stratified by participant's exposure to X-rays (52\% orthopaedics, $35 \%$ cardiologists and $13 \%$ radiologists) or not (72\% orthopaedics, $20 \%$ cardiologists and $8 \%$ radiologists; $p=0.498$ ). Cancer was a recognised as important risk for both professionally exposed physicians and medically exposed patients by $97 \%$ of respondents, independently from the personal exposure to X-rays or not. Non-cancer effects 
(including cataracts, reproductive and neurodegenerative effects) were all recognised as a potential harm by $91 \%$ of respondents, slightly higher percentage within physicians exposed to X-rays $(94 \%)$ than in those not $(90 \% ; p=0.435)$. Moreover, while $98 \%$ of the exposed to X-rays used them in their regular clinical practice, also $51 \%$ of those not classified as exposed declared their use. Figure 1(A) shows perception of chest X-ray equivalents needed for an abdomen computed tomography (CT) scan, also stratified by personal professional exposure: overall, $63 \%$ of respondents correctly identified a corresponding dose of 500 chest X-rays equivalents. No difference in the answer was found between exposed and unexposed operators as shown in Figure 1(B). Among exposed patients, percentage of cancer due to medical X-rays was correctly identified as $5-10 \%$ only by $17 \%$ of responders (Figure $2(\mathrm{~A})$ ), slightly lower within physicians exposed to X-rays $(15 \%)$ than in those not $(18 \% ; p=0.704)$ (Figure 2(B)). In addition, the 3-4 fold higher cancer risk in children compared to adults was widely recognised (95\% of respondents), independently from the personal professional exposure to X-rays $(93 \%)$ or not $(95 \% ; p=0.767)$. Focussing on professionally exposed physicians, the risk of left side brain tumour in interventional cardiologists was known to 58\% of responders, more commonly within physicians exposed to X-rays $(68 \%)$ than in those not $(55 \% ; p=0.081)$. Finally, the European directive (Euratom 2013/59) regulating the mandatory record/report of doses from radiological procedures since February 2018, was known to $45 \%$ of responders, more widely within those exposed to X-rays (64\%) than in those not $(39 \% ; \mathrm{p}<0.001)$.

\section{Discussion}

Our study shows that the majority of physicians tested in the present survey showed a satisfactory level of awareness of doses of common examinations, a correct perception of individual and population radiologic risk associated with radiation exposure and satisfactory 
awareness of the main regulatory aspects concerning the use of radiation in clinical practice. Nevertheless, there is ample room for improvement in knowledge in this key aspect of riskbenefit assessment of all medical imaging techniques. In comparison with previous studies, the radiation awareness was consistently high in our population, with a striking improvement over previous survey testing radiation awareness in Italian cardiologists in 2005 [2] or interventional radiologists in US in 2010 [3]. The reasons for this diffuse rise in awareness of radiological risk are many. In the last 10 years, major scientific societies released position papers on radiological risk emphasising the need to raise radiological awareness in all stakeholders (patients, doctors and industry) and to create a culture of respect for radiation hazard $[1,4]$. The medical core curriculum was restructured with the blueprints of certification and recertification for cardiologists and interventional cardiologists specifying radiation safety subject matter $[5,6]$. The industry has found innovative solutions for reducing several folds of the dose per examination in all fields of medical imaging from cardiac CT to nuclear perfusion imaging up to near zero fluoroscopy in electrophysiology [7-9]. The scientific community is devoting major effort in defining cancer and non-cancer effects of medical radiation with large cohorts of exposed doctors and patients with a combined population and molecular epidemiology approach [10]. The net result of this concerted effort is an increased knowledge witnessed by the current survey results.

\section{Conclusion}

Prescribers and practitioners (mostly cardiologists) working in Italy are largely aware of the cancer and non-cancer risks of medical radiation use, regardless of their subspecialty background. As for the anti-smoking, anti-alcohol, anti-obesity campaigns, medical community should plan more information about harmful effects of ionising radiation, since a risk-awareness may lead to a risk-reduction. On the other hand, physicians ordering and 
performing X-rays should ensure that exposure is as low as reasonably achievable without sacrificing quality of care. Awareness of risks remains the best 'protection' against radiation exposure. 


\section{References}

1.

Picano E, Vañó E, Rehani MM, et al. The appropriate and justified use of medical radiation in cardiovascular imaging: a position document of the ESC Associations of cardiovascular imaging, percutaneous cardiovascular interventions and electrophysiology. Eur Heart J. 2014;35(10):665-672.

2. Correia MJ, Hellies A, Andreassi MG, et al. Lack of radiological awareness among physicians working in a tertiary-care cardiological centre. Int J Cardiol. 2005;103(3):307-311.

3. Miller DL, Vañó E, Bartal G, et al. Occupational radiation protection in interventional radiology: a joint guideline of the Cardiovascular and Interventional Radiology Society of Europe and the Society of Interventional Radiology. Cardiovasc Intervent Radiol. 2010;33(2):230-239.

4. Hirshfeld JW, Jr, Ferrari VA, Bengel FM, et al. 2018 ACC/HRS/NASCI/SCAI/SCCT expert consensus document on optimal use of ionizing radiation in cardiovascular imaging-best practices for safety and effectiveness, part 2: radiological equipment operation, dose-sparing methodologies, patient and medical personnel protection: a report of the American college of cardiology task force on expert consensus decision pathways. J Am Coll Cardiol. 2018;71(24):2829-2855.

5.

Kim C, Vasaiwala S, Haque F, et al. Radiation safety among cardiology fellows. Am J Cardiol. 2010;106(1):125-128.

6.

Roberts EB, Peet DJ. Radiation protection training for cardiologists in the era of multiple imaging techniques and complex interventions. BJR. 2016;89(1067):20160248. 
policies on reducing exposure to ionizing radiation from medical imaging: a systematic review. J Am Coll Radiol. 2015;12(12):1434-1445.

8.

$$
\text { Anselmino M, Sillano D, Casolati D, et al. A new }
$$

electrophysiology era: zero fluoroscopy. J Cardiovasc Med. 2013;14(3):221-227.

9.

Giaccardi M, Del Rosso A, Guarnaccia V, et al. Near-zero x-ray

in arrhythmia ablation using a 3-dimensional electroanatomic mapping system: a multicenter experience. Heart Rhythm. 2016;13(1):150-156.

10. Hirshfeld JW, Jr, Balter S, Brinker JA, et al. ACCF/AHA/HRS/SCAI clinical competence statement on physician knowledge to optimize patient safety and image quality in fluoroscopically guided invasive cardiovascular procedures: a report of the American College of Cardiology Foundation/American Heart Association/American College of Physicians Task Force on Clinical Competence and Training. Circulation. 2005;111(4):511-532. 
Table 1. Full details of the 10 step voluntary web based survey and number of receipts.

\begin{tabular}{|c|c|}
\hline Question & number of answers \\
\hline $\begin{array}{l}\text { 1. Your main activity is: } \\
\text { - clinical cardiology - interventional cardiology - oncology - hemodynamics } \\
\text { - electrophysiology - cardiac stimulation - internal medicine - diabetology - other }\end{array}$ & 2023 \\
\hline $\begin{array}{l}\text { 2. The Physician that mostly prescribed x-rays are: } \\
\text { - orthopaedics - cardiologists - radiologists - nephrologists }\end{array}$ & 1962 \\
\hline $\begin{array}{l}\text { 3. X-ray exposure relates to cancer both in patients than in operators: } \\
\text { - yes - no }\end{array}$ & 1942 \\
\hline $\begin{array}{l}\text { 4. Other than cancer, } \mathrm{x} \text {-ray may cause: } \\
\text { - reproductive effects - cataracts - neurodegeneration - all these conditions }\end{array}$ & 1941 \\
\hline $\begin{array}{l}\text { 5. Do you use x-rays in your clinical practice? } \\
- \text { yes - no }\end{array}$ & 1940 \\
\hline $\begin{array}{l}\text { 6. How many chest } x \text {-rays are equivalent to the dose needed for an abdomen } \\
\text { Computed Tomography? } \\
-1 \text { chest } x \text {-ray }-10 \text { chest } x \text {-rays }-500 \text { chest } x \text {-rays }-5000 \text { chest } x \text {-rays }\end{array}$ & 1901 \\
\hline $\begin{array}{l}\text { 7. The percentage of cancer due to medical } x \text {-rays is: } \\
-1 \%-2 \%-3 \%-5-10 \%\end{array}$ & 1883 \\
\hline $\begin{array}{l}\text { 8. Operators exposed to x-rays may more easily suffer from left sided brain } \\
\text { tumors? } \\
\text { - yes - no }\end{array}$ & 1882 \\
\hline $\begin{array}{l}\text { 9. Are you aware of the European Directive } 2013 / 59 / \text { EURATOM that requires } \\
\text { reporting x-ray dose related to a medical diagnostic investigation or therapy? } \\
\text { - yes }- \text { no }\end{array}$ & 1861 \\
\hline $\begin{array}{l}\text { 10. Is it documented that the risk of cancer related to x-ray exposure if } 4 \text {-fold } \\
\text { higher in children than in adults? } \\
\text { - yes - no }\end{array}$ & 1861 \\
\hline
\end{tabular}


Table 2. Main clinical activity of physicians approaching the web based survey $(n=2023)$.

\begin{tabular}{|l|c|}
\hline Main clinical activity & Percentage \\
\hline clinical cardiology & $40 \%$ \\
\hline interventional cardiology & $10 \%$ \\
\hline hemodynamics & $6 \%$ \\
\hline electrophysiology & $9 \%$ \\
\hline cardiac stimulation & $7 \%$ \\
\hline internal medicine & $19 \%$ \\
\hline $\begin{array}{l}\text { oncology } \\
\text { diabetology }\end{array}$ & $2 \%$ \\
\hline $\begin{array}{l}\text { others (anesthesiology, general practitioner, } \\
\text { nephrology, geriatrics, emergency medicine, } \\
\text { vascular surgery) }\end{array}$ & $5 \%$ \\
\hline
\end{tabular}


Figure 1. (A) Perception of chest X-ray equivalents needed for an abdomen CT scan; (B) Data also stratified by personal exposure 1 chest $\mathrm{X}$-ray (A):3\%, (B):2\%-4\% respectively. 10 chest X-rays (A):27\%, (B):23\%-28\% respectively. 500 chest X-rays (A):63\%, (B):64\%-62\% respectively. 5000 chest $\mathrm{X}$-rays (A):7\%, (B):7\%-6\% respectively.

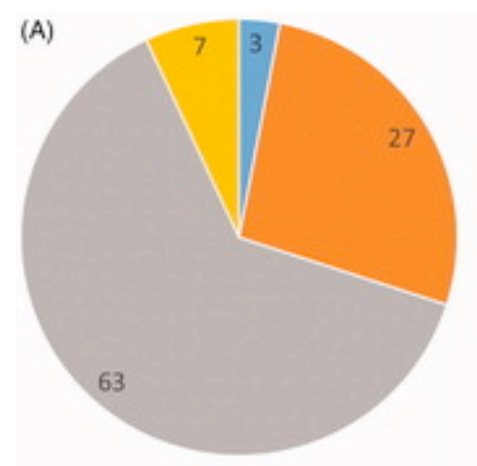

(B)
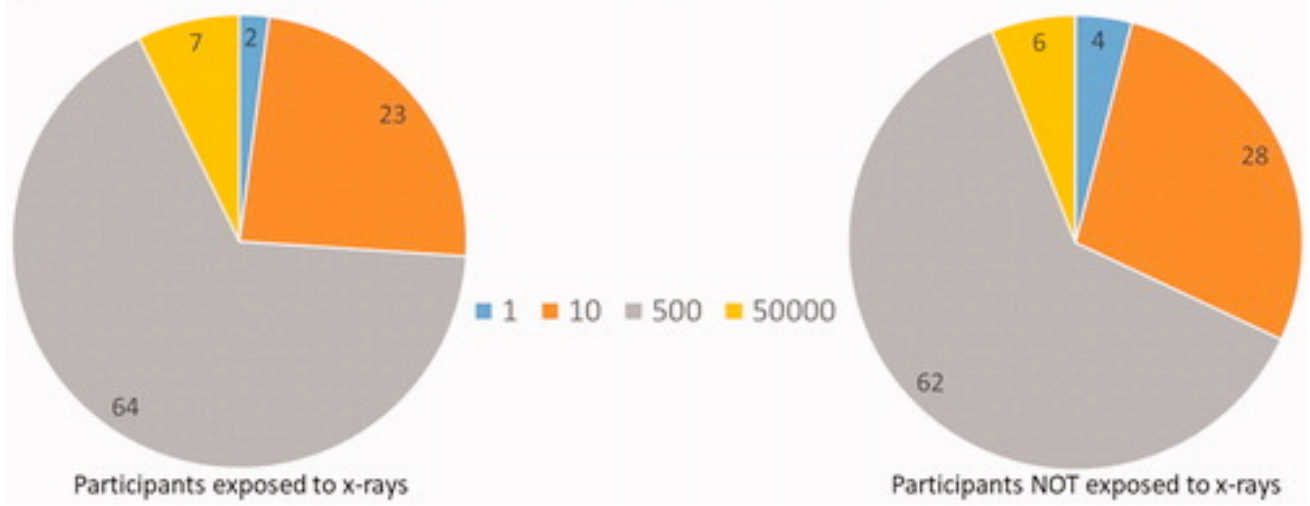
Figure 2. (A) Participant's opinion on the percentage of cancers due to medical X-rays; (B) Data also stratified by personal exposure. 1\% (A):26\%, (B):35\%-22\% respectively. 2\% (A):28\%, (B):28\%-28\% respectively. 3\% (A):29\%, (B):22\%-32\% respectively. 5-10\% (A):17\%, (B):15\%-18\% respectively.
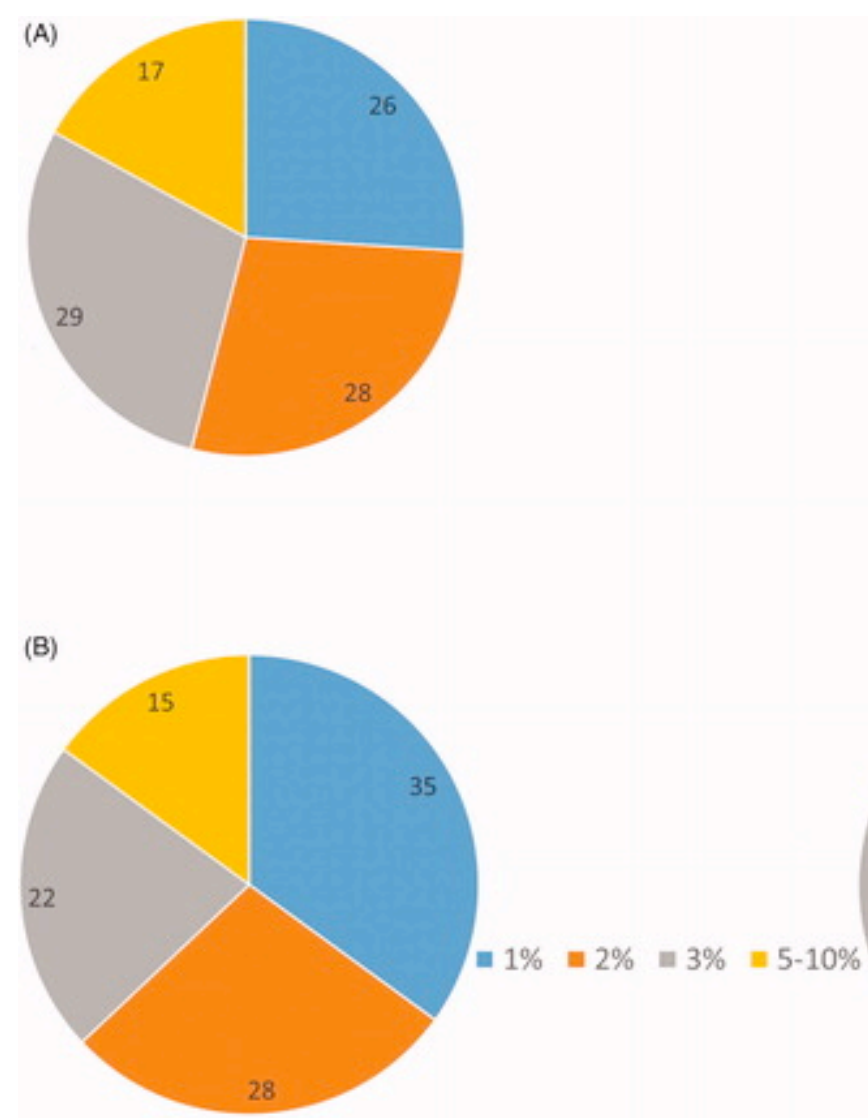

Participants exposed to $\mathrm{x}$-rays $n=489$

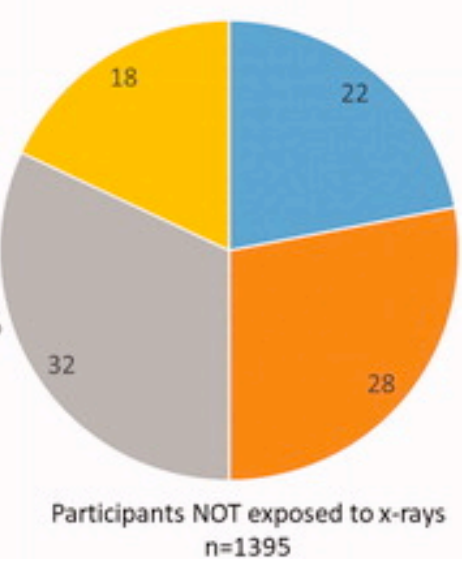

